\title{
Drug designed to raise HDL levels falls down
}

O

n December 2, 2006, Pfizer halted the development of torcetrapib, the drug that was once considered by the company and pharmaceutical analysts as the most promising drug in its pipeline.

The development of torcetrapib as a treatment for heart disease was brought to an end because preliminary data from a 15,000patient phase III clinical trial indicated that individuals receiving the drug had higher risks of death and heart failure than did individuals not receiving the drug.

The trial was organized such that 7,500 patients taking torcetrapib and the statin Lipitor (atorvastatin) were compared with 7,500 patients taking Lipitor alone. It was hoped that the drug combination would decrease the risk of coronary heart disease and stroke more than Lipitor alone. However, the independent panel of experts monitoring the trial noted that 82 patients receiving the drug combination had died compared with only 51 receiving Liptor alone and advised Pfizer to halt the trial, which it did immediately.

The news came as a surprise to many people, including Jeffrey L. Kindler, the new chief executive officer of Pfizer, who only days earlier at a meeting for investors was quoted as saying, "This will be one of the most important compounds of our generation" (1). Similarly, Daniel J. Rader, a preventative cardiovascular specialist at the University of Pennsylvania who was senior author on the first published manuscript on torcetrapib, told the JCI, "Although there were legitimate questions about whether cholesterol ester transfer protein (CETP) inhibition with torcetrapib would substantially reduce atherosclerosis progression and cardiovascular events, I personally did not anticipate that the drug would result in increased cardiovascular events and mortality."

The reason that many people, such as Rader, had high hopes for torcetrapib is that it substantially increases levels of HDL, which is generally considered "good cholesterol," and decreases levels of LDL, which is generally considered "bad cholesterol." Low levels of HDL are associated with an increased risk of developing coronary heart disease, and so it had been hoped that combining torcetrapib with
Lipitor, which mainly works (as all statins do) by decreasing LDL levels, would further decrease the risk of coronary heart disease and strokes.

Torcetrapib, in development since 1990, works by inhibiting CETP, which transfers cholesterol esters from HDL to other lipoproteins. One early indication that developing such drugs would increase

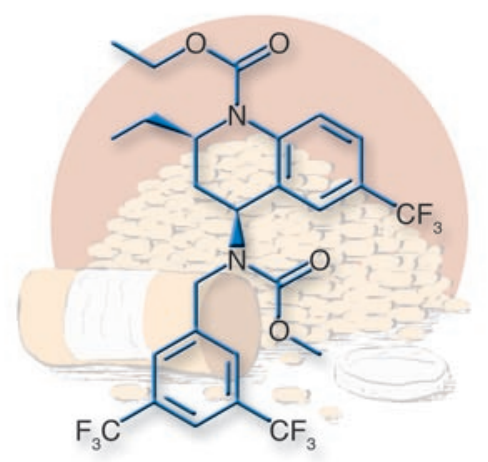

Figure 1

The structure of torcetrapib, the heart disease drug whose development by Pfizer was halted in early December 2006.

HDL levels and therefore be useful for treating coronary heart disease was the observation that humans with a defect in the gene encoding CETP have increased levels of HDL (2). However, a later study, published in the JCI, showed that despite an increase in HDL levels, Japanese-American men with a defect in CETP had an increased risk of coronary heart disease (3). Alan Tall at Columbia University, who was the senior author on both these studies $(2,3)$, told the JCI that despite the observation of increased risk of coronary heart disease in Japanese-American men with a defect in CETP (3), further genetic studies "showed no clear increase or decrease in coronary heart disease [linked to a defect in CETP].”

It is currently unclear why torcetrapib had such a devastating effect, and the future development and utility of other CETP inhibitors depends on the answer to this question. One possibility lies in the observation that torcetrapib has been shown to actually increase systolic blood pressure. Other CETP inhibitors in devel- opment have not been reported to have this effect, so it might not be the end of the road for all CETP inhibitors.

Additional possible explanations for the failure of torcetrapib include other potential off-target effects of torcetrapib, insufficient blockage of CETP function, and unwanted interactions between torcetrapib and Lipitor. It might also be that the strategy of increasing HDL levels per se is not enough, and some researchers believe that it is the function of HDL that needs to be improved rather than its levels (4). Indeed, some researchers are actively pursuing this as an approach to decrease the risk of individuals developing coronary heart disease.

Whatever the reason for the failure of torcetrapib, coronary heart disease remains the number one killer in the United States, and as Tall remarked, "[Statins] have not solved the problem of coronary heart disease; we still need more research and to continue the development of new drugs to tackle this problem.”

As for Pfizer, analysts believe that the loss of torcetrapib from its pipeline is a major blow to the company, which was hoping that the combination of torcetrapib and Lipitor would extend the life of Lipitor past the expiry of its patent protection in 2011. Despite this, and the upcoming loss of patent protection on other high-income drugs such as Viagra, Kindler told the New York Times that the company still expected to report higher profits in both 2007 and 2008, mainly due to cost cutting (5).

\section{Karen Honey}

1. Berenson, A. 2006 December 1. Pfizer likely to seek approval of new heart drug in 2007. The New York Times. http://query.nytimes.com/gst/fullpage. html?sec $=$ health\&res $=9$ C00EFDB1E3EF932A357 51C1A9609C8B63.

2. Inazu, A., et al. 1990. Increased high-density lipoprotein levels caused by a common cholesterylester transfer protein gene mutation. N. Engl. J. Med. 323:1234-1238.

3. Zhong, S., et al. 1996. Increased coronary heart disease in Japanese-American men with mutation in the cholesteryl ester transfer protein gene despite increased HDL levels. J. Clin. Invest. 97:2917-2923.

4. Rader, D.J. 2006. Molecular regulation of HDL metabolism and function: implications for novel therapies. J. Clin. Invest. 116:3090-3100. doi:10.1172/JCI30163.

5. Berenson, A. 2006 December 4. End of drug trial is a big loss for Pfizer. The New York Times. http://www. nytimes.com/2006/12/04/health/04pfizer.html. 